\title{
Lipid Deposition in Various Sites of the Skeletal Muscles and Liver Exhibits a Positive Correlation with Visceral Fat Accumulation in Middle-aged Japanese Men with Metabolic Syndrome
}

\author{
Shin-ichiro Taira ${ }^{1}$, Michio Shimabukuro ${ }^{2}$, Moritake Higa ${ }^{3}$, Kouichi Yabiku ${ }^{1}$, \\ Chisayo Kozuka ${ }^{1}$, Rei Ueda ${ }^{1}$, Sumito Sunagawa ${ }^{1}$, Yuzuru Ohshiro ${ }^{1}$, Mototsugu Doi ${ }^{1}$, \\ Toyotaka Nanba ${ }^{1}$, Eriko Kawamoto ${ }^{1}$, Yoshiro Nakayama ${ }^{1}$, Hideaki Nakamura ${ }^{1}$, Takako Iha ${ }^{1}$, \\ Sawako Nakachi ${ }^{1}$, Takeaki Tomoyose ${ }^{1}$, Tomomi Ikema ${ }^{1}$, \\ Ken Yamakawa ${ }^{1}$ and Hiroaki Masuzaki ${ }^{1}$
}

\begin{abstract}
Objective In addition to excess visceral fat, lipid deposition in the liver and skeletal muscle has been implicated in the pathophysiology of type 2 diabetes and metabolic syndrome. This study was designed to explore the relationship between hepatic and muscular lipid deposition and visceral fat accumulation in 105 middleaged men with metabolic syndrome.

Methods Abdominal computed tomography (CT) was used to simultaneously evaluate the visceral fat area (VFA) and CT Hounsfield unit (HU) values of three different portions of skeletal muscle and the liver.

Results A significant inverse correlation was observed between the VFA and the CT HU values of the iliopsoas muscle, back muscle, rectus abdominis muscle and liver. Three types of interventions, i.e., lifestyle modification and treatment with antidiabetic drugs, such as Pioglitazone or Miglitol, caused significant decreases in visceral fat accumulation. The extent of lipid deposition in the liver was strongly correlated with the levels of glucose-lipid metabolic markers, which decreased significantly following Pioglitazone treatment. On the other hand, the amount of lipid deposition in the three skeletal muscles and the liver did not decrease after Miglitol treatment.

Conclusion Visceral fat accumulation is accompanied by excess lipid deposition in skeletal muscle and the liver in patients with metabolic syndrome. The CT-based simultaneous, concise evaluations of ectopic lipid deposition and visceral fat mass used in the present study may provide unique information for assessing cardiometabolic risks and the therapeutic impact in patients with diabetes-obesity syndrome.
\end{abstract}

Key words: metabolic syndrome, diabetes, visceral fat, ectopic lipid deposition, computed tomography

(Intern Med 52: 1561-1571, 2013)

(DOI: 10.2169/internalmedicine.52.0521)

\section{Introduction}

In addition to visceral fat accumulation $(1,2)$, ectopic lipid deposition, especially in the liver and skeletal muscle, has been implicated in the pathophysiology of diabetes, insulin resistance and dyslipidemia (3). Of note, Japanese patients with metabolic syndrome exhibit a relatively lower degree of obesity than Caucasians (4). However, the prevalence of diabetes among the Japanese population is compa-

\footnotetext{
${ }^{1}$ Division of Endocrinology, Diabetes and Metabolism, Hematology, Rheumatology (Second Department of Medicine), Graduate School of Medicine, University of the Ryukyus, Japan, ${ }^{2}$ Department of Cardio-Diabetes Medicine, The University of Tokushima Graduate School of Health Biosciences, Japan and ${ }^{3}$ Diabetes and Life-style Related Disease Center, Tomishiro Central Hospital, Japan

Received for publication March 5, 2013; Accepted for publication March 24, 2013

Correspondence to Dr. Hiroaki Masuzaki, hiroaki@med.u-ryukyu.ac.jp
} 
rable to that observed in the United States, thus leading to the speculation that excessive energy intake by Japanese individuals may exaggerate ectopic lipid deposition due to their limited capacity to store subcutaneous fat tissue (5).

Animal experiments have demonstrated that excess accumulation of lipids in pancreatic islets is associated with progressive beta cell apoptosis, which results in impaired secretion of insulin (6). When fed a high-fat diet, transgenic mice overexpressing the leptin receptor $(\mathrm{Ob}-\mathrm{R})$ exclusively in adipose tissue under a genetically obese and diabetic $d b / d b$ background exhibit marked weight reduction compared to $d b / d b$ mice per se. In contrast, lipid deposition in the liver, cardiac muscle and pancreas is pronounced, which aggravates the severity of diabetes in transgenic animals (7). Agedependent declines in the mitochondrial function and increases in intramyocellular lipid content have also been reported (8). In humans, lifestyle modification markedly decreases intramyocellular lipid deposition in association with concomitant increases in insulin-mediated glucose uptake in muscles (9).

To the best of our knowledge, there are no reports describing the association between visceral fat accumulation and lipid deposition in the liver or skeletal muscle in a large number of patients with metabolic syndrome. Therefore, the present study was designed to investigate the metabolic link between lipid deposition, particularly in skeletal muscles and the liver, and visceral fat accumulation in relation to lifestyle and pharmacological interventions. Recently, proton magnetic resonance spectroscopy ( $\left.{ }^{1} \mathrm{H}-\mathrm{MRS}\right)$ has been employed to precisely evaluate ectopic lipid accumulation. Because the technique is expensive and elaborate, however, its use is not yet prevalent in clinics. In this context, we attempted to establish a concise method for evaluating ectopic lipid deposition using abdominal computed tomography (CT) scanning.

\section{Materials and Methods}

\section{Subjects}

Our study included patients who visited Tomishiro Central Hospital (Okinawa prefecture, Japan) via self-referral or recommendation for further checkup by local health care institutions. In the present study, we performed an ad hoc analysis using the pooled database of 332 men who agreed to participate in interventional trials performed at Tomishiro Central Hospital. The results of some parts of these interventional trials have recently been published (10), while other parts are currently in the process of manuscript preparation. Subjects taking antidyslipidemic or antidiabetic drugs were completely excluded. Furthermore, no drugs were administered to subjects recruited during the observation period. The convergence of metabolic abnormalities was evaluated during medical checkups. According to the current definition of metabolic syndrome in Japan $(11,12)$, the presence of abdominal obesity (a waist circumference of $\geq 85 \mathrm{~cm}$ for men and $\geq 90 \mathrm{~cm}$ for women) is essential for making a diagnosis of metabolic syndrome. In the present study, metabolic syndrome was defined according to the 2005 International Diabetes Federation (IDF) guidelines (13), which include the presence of at least two of the following four factors: (1) elevated triglycerides (TG) $(\geq 150 \mathrm{mg} / \mathrm{dL}(1.7 \mathrm{mmol} / \mathrm{L})),(2)$ reduced high-density lipoprotein (HDL) cholesterol $(<40$ $\mathrm{mg} / \mathrm{dL}(1.03 \mathrm{mmol} / \mathrm{L})$ in men and $<50 \mathrm{mg} / \mathrm{dL}(1.29 \mathrm{mmol} /$ L) in women), (3) elevated blood pressure (a systolic blood pressure of $\geq 130 \mathrm{mmHg}$ or a diastolic blood pressure of $\geq 85$ $\mathrm{mmHg}$ or both) and (4) elevated fasting plasma glucose (FPG) $(\geq 100 \mathrm{mg} / \mathrm{dL}(5.6 \mathrm{mmol} / \mathrm{L}))$. The present study was approved by the ethical committee for human research at Tomishiro Central Hospital (November 16, 2011. No. 104).

\section{Study 1: Relationship between ectopic lipid deposi- tion and metabolic parameters}

Evidence regarding the association between excessive lipid deposition in nonadipose tissue, also known as ectopic lipid deposition, and metabolic derangement has been accumulating (14). In Study 1, we focused on ectopic lipid deposition in the skeletal muscles and liver. We analyzed 105 men under 60 years of age who underwent abdominal CT before receiving various types of clinical interventions. The study included 105 middle-aged men (age: 49.0 \pm 8.0 years) with metabolic syndrome who were diagnosed using the IDF criteria described above. At the umbilical level, the CT Hounsfield unit (HU) values of three different regions of skeletal muscle (the iliopsoas muscle, back muscle and rectus abdominis muscle) and the visceral fat area (VFA) were simultaneously measured using unenhanced CT. On the CT slices obtained at the umbilical level, the adipose tissue area located in the peritoneal cavity was defined as the VFA and the adipose tissue area underlying the skin layer was defined as the subcutaneous fat area (SFA). Using the abdominal CT images in the pooled database, we measured the CT values of the iliopsoas muscle, back muscle, rectus abdominis muscle and liver. In the present study, the CT HU value was used to assess the relative extent of ectopic lipid deposition, as described below. Anthropometric and metabolic parameters were analyzed as previously reported (Table 1 (15)).

\section{Study 2: Effects of lifestyle modification and phar- maceutical interventions on ectopic lipid deposition and fuel metabolism}

The impact of lifestyle modification and pharmacological interventions on ectopic lipid deposition and glucose-lipid metabolism was investigated by examining 68 men between 32 and 76 years of age. Before the intervention, all participants received guidance for lifestyle modification. The subjects followed a standard diet $(25 \mathrm{kcaL} / \mathrm{kg}+0-200 \mathrm{kcaL})$ and engaged in regular exercise by walking approximately 10,000 steps per day or approximately one hour per day for three to five days per week during the study period. In Study 2, we analyzed 181 subjects who received interventions with either lifestyle modification alone (104 cases), 
Table 1. Patient Characteristics (Study 1)

\begin{tabular}{|c|c|}
\hline Age (years) & $49.0 \pm 8.0$ \\
\hline Body weight $(\mathrm{kg})$ & $78.2 \pm 9.9$ \\
\hline BMI $\left(\mathrm{kg} / \mathrm{m}^{2}\right)$ & $27.8 \pm 3.4$ \\
\hline Waist circumference $(\mathrm{cm})$ & $93.9 \pm 7.4$ \\
\hline $\operatorname{VFA}\left(\mathrm{cm}^{2}\right)$ & $185.0 \pm 58.3$ \\
\hline $\mathrm{SFA}\left(\mathrm{cm}^{2}\right)$ & $177.8 \pm 66.6$ \\
\hline $\mathrm{FPG}(\mathrm{mg} / \mathrm{dL})$ & $115.0 \pm 23.0$ \\
\hline Fasting insulin $(\mu \mathrm{U} / \mathrm{mL})$ & $13.0 \pm 6.8$ \\
\hline HOMA-IR & $3.6 \pm 2.0$ \\
\hline НОМА- $\beta$ & $99.7 \pm 58.7$ \\
\hline HbAlc $(\%)$ & $7.0 \pm 1.3$ \\
\hline T-Cho (mg/dL) & $210.0 \pm 35.8$ \\
\hline HDL-C (mg/dL) & $47.0 \pm 10.9$ \\
\hline $\mathrm{TG}(\mathrm{mg} / \mathrm{dL})$ & $213.0 \pm 215.4$ \\
\hline $\operatorname{AST}(\mathrm{IU} / \mathrm{L})$ & $30.0 \pm 18.7$ \\
\hline $\operatorname{ALT}(\mathrm{IU} / \mathrm{L})$ & $45.0 \pm 29.5$ \\
\hline$\gamma$-GTP (IU/L) & $82.0 \pm 83.6$ \\
\hline $\mathrm{UA}(\mathrm{mg} / \mathrm{dL})$ & $6.3 \pm 1.3$ \\
\hline $\mathrm{CRP}(\mathrm{mg} / \mathrm{dL})$ & $0.2 \pm 0.2$ \\
\hline $\mathrm{FFA}(\mathrm{mmol} / \mathrm{L})$ & $0.5 \pm 0.2$ \\
\hline $\mathrm{SBP}(\mathrm{mmHg})$ & $140.0 \pm 15.3$ \\
\hline $\mathrm{DBP}(\mathrm{mmHg})$ & $87.0 \pm 9.7$ \\
\hline Pulse (beats/min) & $78.0 \pm 12.2$ \\
\hline CT HU value of iliopsoas muscle & $44.2 \pm 5.9$ \\
\hline CT HU value of back muscle & $41.8 \pm 8.2$ \\
\hline CT HU value of rectus abdominis muscle & $28.2 \pm 14.1$ \\
\hline CT HU value of liver & $50.2 \pm 11.3$ \\
\hline CT HU value of spleen & $47.8 \pm 5.1$ \\
\hline Iliopsoas muscle area $\left(\mathrm{cm}^{2}\right)$ & $1,322.3 \pm 277.2$ \\
\hline Back muscle area $\left(\mathrm{cm}^{2}\right)$ & $2,775.4 \pm 590.7$ \\
\hline Rectus abdominis muscle area $\left(\mathrm{cm}^{2}\right)$ & $602.0 \pm 149.7$ \\
\hline
\end{tabular}

The plus-minus values indicate the mean $\pm \mathrm{SD}$.

VFA : Visceral fat area, SFA : Subcutaneous fat area, FPG : Fasting plasma glucose, HDL-C: High-density lipoprotein cholesterol, TG: Triglyceride, AST: Aspartate 2-oxoglutarate aminotransferase, ALT: Alanine 2-oxoglutarate aminotransferase, UA: Uric acid, CRP: C-reactive protein, HbA1c: Glycosylated hemoglobin, T-Cho: Total cholesterol, FFA: Free fatty acid, $\gamma$-GTP: Gamma-glutamyl transpeptidase, SBP: Systolic blood pressure, DBP: Diastolic blood pressure, BMI: Body mass index

The body mass index is the weight in kilograms divided by the square of the height in meters.

The value for $\mathrm{HbAlc}(\%)$ is estimated as the NGSP equivalent value (\%) calculated according to the formula HbA1c (\%) $=\mathrm{HbAlc}(\mathrm{JDS})(\%)+0.4 \%$, considering the relational expression of HbA1c (JDS)(\%) measured based on the previous Japanese standard substance and measurement methods and HbA1c (NGSP) (15).

JDS: Japan Diabetes Society, NGSP: National Glycohemoglobin Standardization Program

lifestyle modification with Pioglitazone treatment (39 cases) or lifestyle modification with Miglitol treatment (38 cases). The baseline characteristics were roughly similar between the three groups.
Next, we performed an ad hoc analysis of 68 men who underwent abdominal CT before and after receiving various types of clinical interventions, a group who underwent lifestyle modification alone ( 28 cases), a group who underwent lifestyle modification with Pioglitazone treatment (24 cases) and a group who underwent lifestyle modification with Miglitol treatment (16 cases). Briefly, Group A ( $n=28$; age: $55 \pm 11.4$ years) maintained the lifestyle modifications for three months. Group B ( $n=24$; age: $55 \pm 9.0$ years) received treatment with Pioglitazone (30 mg/day) for three months. Group C ( $\mathrm{n}=16$; age: $58 \pm 9.7$ years) received treatment with Miglitol (150 mg/day) for three months. Before and after the treatment period, the amount of ectopic lipid deposition and the anthropometric and metabolic parameters were analyzed, as in Study 1. The clinical characteristics of the Study 2 participants are summarized in Table 2.

\section{Assessment of the CT HU values of the nonadipose tissues}

\section{(1) Assessment of the CT HU values of the three types of skeletal muscles}

CT scans of lipid deposition in skeletal muscle, including the iliopsoas muscle, back muscle and rectus abdominis muscle, performed at the umbilical level usually result in the detection of patchy or diffuse areas (16). Commonly, a region of interest (ROI) is determined to evaluate the average CT HU value (17). Notably, skeletal muscles are known to exhibit wide regional differences in the density of muscle fibers $(16,18)$, and the CT HU value of the muscle greatly depends on the ROI location. Therefore, in the present study, ROIs were not used. Instead, minor modifications were made to a previously described method (19), according to which cross sections of the iliopsoas muscle, back muscle and rectus abdominis muscle were outlined at the umbilical level using a cursor. The CT HU value of the region surrounded by the line was employed as the average CT HU value of the respective skeletal muscle, and the values obtained on each side were averaged to obtain the representative CT HU value. A lower CT HU value reflects increased lipid deposition in each tissue.

\section{(2) Assessment of the CT HU values of the liver}

Similar to the results of the analyses of skeletal muscles, lipid deposition in the liver is usually detected in a diffuse or patchy pattern (20). Contrary to the evaluations of skeletal muscles, ROIs were employed to assess the CT HU values of the liver in this study. To carefully prevent contamination of blood vessels, ROIs broader than $100 \mathrm{~mm}^{2}$ were measured in the liver parenchyma (two sites in the right lobe and one site in the left lobe) (21). The average CT HU value assessed according to this method exhibited a significant inverse correlation with the degree of fatty liver, as described in the Results section. 
Table 2. Clinical Parameters Measured before and after Three Months of Intervention

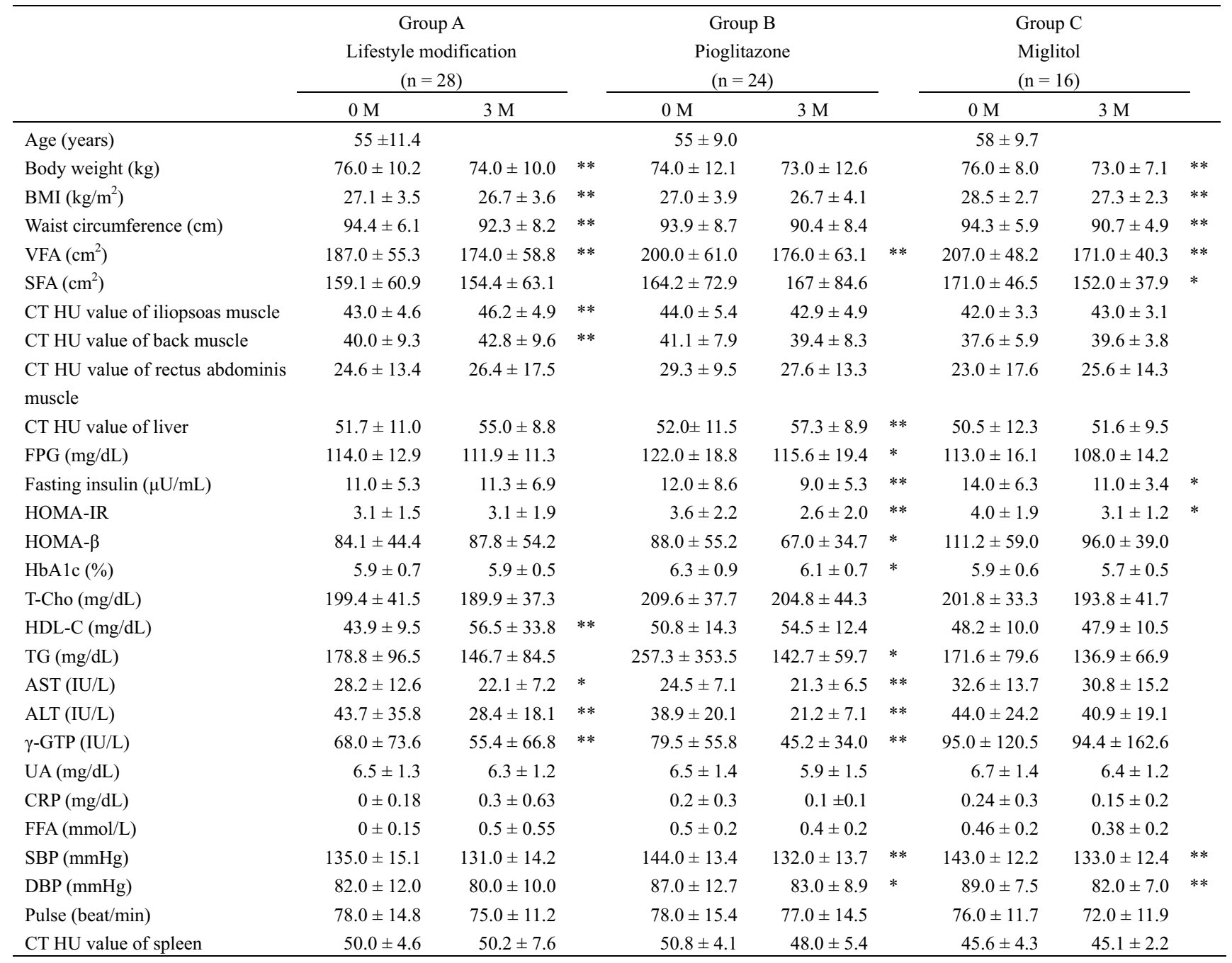

The plus-minus values indicate the mean $\pm \mathrm{SD}$.

The body mass index is the weight in kilograms divided by the square of the height in meters.

$* \mathrm{p}<0.05,{ }^{* *} \mathrm{p}<0.01$ vs. 0M

VFA: Visceral fat area, SFA: Subcutaneous fat area, FPG: Fasting plasma glucose, HDL-C: High-density lipoprotein cholesterol, TG: Triglyceride, AST: Aspartate 2-oxoglutarate aminotransferase, ALT: Alanine 2-oxoglutarate aminotransferase, UA: Uric acid, CRP: C-reactive protein, HbA1c: Glycosylated hemoglobin, T-Cho: Total cholesterol, FFA: Free fatty acid, $\gamma$-GTP: Gamma-glutamyl transpeptidase, SBP: Systolic blood pressure, DBP: Diastolic blood pressure, BMI: Body mass index

\section{Statistical analyses}

The values are expressed as the mean \pm standard deviation (SD). The differences were analyzed using paired Student's $t$-test or the Wilcoxon signed-rank test as appropriate. Pearson's correlation coefficients and Spearman's correlation coefficients were used where applicable. Values that were not distributed normally were transformed into logarithms (base e) (log) and subsequently analyzed using Pearson's correlation coefficients. Values that were not distributed normally even after being transformed into logarithms (base e) (log) were analyzed using Spearman's correlation coefficients. Multiple regression analyses were applied to determine whether a line of variables was significantly associated with glucose-lipid metabolic markers. Due to the crosssectional nature of the analysis, the associations do not nec- essarily indicate causality. Therefore, we attempted to investigate the potential associations between the changes in the CT HU values of the three skeletal muscles (delta value: 3 months (3M)-0 month (0M)) and changes in the other variables, including VFA (delta value: 3 months (3M)-0 month $(0 \mathrm{M})$ ), during the observation period. A $\mathrm{p}$ value of $<0.05$ was considered to be statistically significant.

\section{Results}

\section{Study 1}

A total of 105 men (age: $49.0 \pm 8.0$ years; BMI: $27.8 \pm 3.4$ $\mathrm{kg} / \mathrm{m}^{2}$; waist circumference: $93.9 \pm 7.4 \mathrm{~cm}$; body weight: $78.2 \pm 9.9 \mathrm{~kg}$ ) were included in the present study. The CT HU values of each muscle were as follows: iliopsoas mus- 


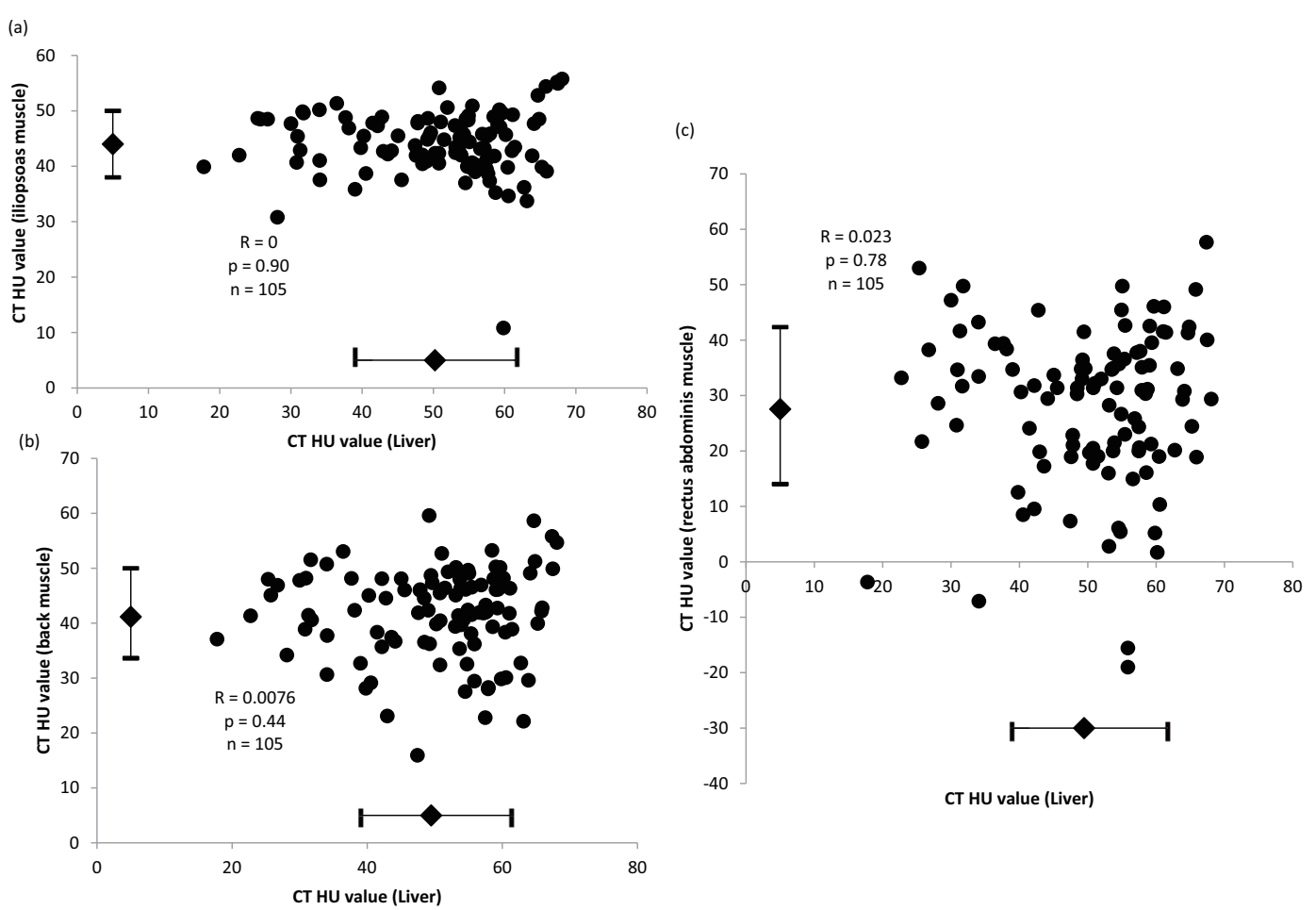

Figure 1. A comparison of the computed tomography (CT) Hounsfield unit (HU) values of the liver and skeletal muscles. No correlations were observed between the CT HU values of the iliopsoas muscle and liver (a), between the CT HU values of the back muscle and liver (b) and between the CT HU values of the rectus abdominis muscle and liver (c). Overall, the CT HU values of the liver exhibited greater variability than those of the skeletal muscles. Among the three skeletal muscles, the CT HU values of the rectus abdominis muscle exhibited the widest variation.

cle: 44.2 $\pm 5.9 \mathrm{HU}$ (95\% confidence interval (CI), 43.1-45.3); back muscle: $41.8 \pm 8.2 \mathrm{HU}$ (95\% CI, 40.2-43.4); and rectus abdominis muscle: $28.2 \pm 14.1 \mathrm{HU}$ (95\% CI, 25.5-30.9). The intrasubject coefficients of variation of the CT HU values of the iliopsoas muscle, back muscle, rectus abdominis muscle and liver were $1.6 \%, 1.1 \%, 4.4 \%$ and $0.7 \%$, respectively $(\mathrm{n}=$ 10). The intersubject coefficients of variation of the CT HU values of the iliopsoas muscle, back muscle, rectus abdominis muscle and liver were $8.7 \%, 5.1 \%, 36.0 \%$ and $25.8 \%$, respectively $(n=5)$.

Notably, there were no correlations between the CT HU values of the three types of skeletal muscles and those of the liver. Overall, the CT HU values of the liver exhibited greater variability than those of the skeletal muscles (Fig. 1). Among the three skeletal muscles, the CT HU values of the rectus abdominis exhibited greater variation (Fig. 1). Importantly, significant inverse correlations were observed between the VFA and the CT HU values of the iliopsoas muscle, back muscle, rectus abdominis muscle ( $\mathrm{r}=-0.27, \mathrm{r}=-0.47$ and $\mathrm{r}=-0.37$, respectively; $\mathrm{p}<0.01)$ and liver $(\mathrm{r}=-0.23, \mathrm{p}<$ 0.05) (Fig. 2). Furthermore, significant inverse correlations were observed between age and the CT HU values of the iliopsoas muscle, back muscle and rectus abdominis muscle $(\mathrm{r}=-0.45, \mathrm{r}=-0.45$ and $\mathrm{r}=-0.41$, respectively; $\mathrm{p}<0.01)$. In contrast, no correlations were observed between the CT HU values of the three skeletal muscles and body weight, waist circumference, SFA, the levels of hepatic enzymes or the levels of glucose-lipid metabolic markers.

Significant positive correlations were observed between the iliopsoas muscle area and BMI, waist circumference and SFA $(r=0.54, r=0.40$ and $r=0.38$, respectively; $p<0.01)$. Similarly, significant positive correlations were observed between the rectus abdominis muscle area and BMI, waist circumference and SFA $(r=0.47, r=0.44$ and $r=0.29$, respectively; $\mathrm{p}<0.01)$. The iliopsoas muscle area was correlated with the levels of fasting insulin, homeostasis model assessment of insulin resistance (HOMA-IR), aspartate 2oxoglutarate aminotransferase (AST) and alanine 2oxoglutarate aminotransferase (ALT) $(r=0.38, \quad r=0.38, \quad r=$ $0.32, \mathrm{p}<0.01 ; \mathrm{r}=0.30, \mathrm{p}<0.05)$. Inverse correlations were observed between the back muscle area and HDL-C and free fatty acid (FFA) ( $r=-0.31$ and $r=-0.36$, respectively; $\mathrm{p}<0.01$ ). However, no correlations were observed between the back muscle area and BMI, waist circumference or SFA.

A significant positive correlation was observed between the iliopsoas muscle area and the CT HU value of the iliopsoas muscle $(\mathrm{r}=0.30, \mathrm{p}<0.01)$. Similarly, a significant positive correlation was observed between the back muscle area and the CT HU value of the back muscle $(\mathrm{r}=0.28, \mathrm{p}<0.01)$. On the other hand, no correlations were observed between the rectus abdominis muscle area and the CT HU value of the rectus abdominis muscle $(\mathrm{r}=0.17, \mathrm{p}=0.07)$.

We next explored the possible associations between the CT HU values and the levels of glucose-lipid metabolic 
(a)

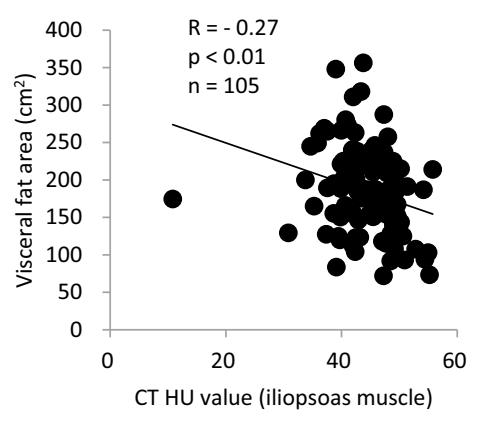

(c)

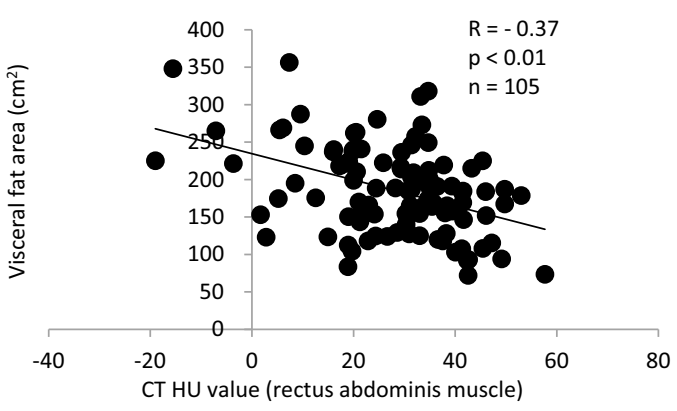

(b)

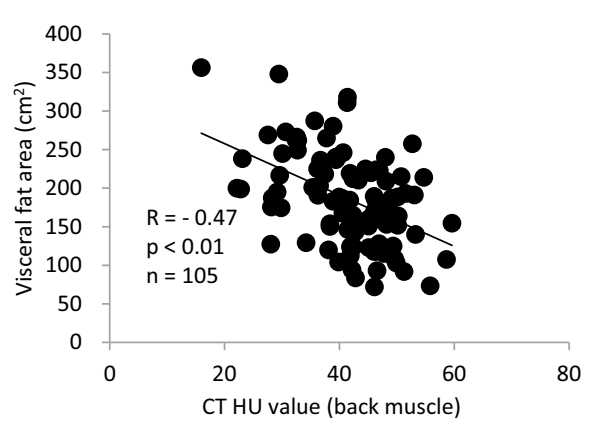

(d)

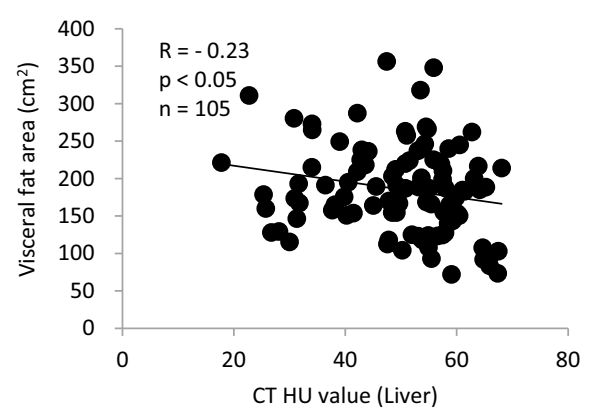

Figure 2. A comparison between the visceral fat area and the CT HU values of the liver and skeletal muscles. Significant inverse correlations were observed between the visceral fat area and the CT HU values of the iliopsoas muscle (a), back muscle (b), rectus abdominis muscle (c) and liver (d).

markers in groups stratified according to BMI. Inverse correlations were observed between the CT HU value of the iliopsoas muscle and the levels of triglycerides and HOMA$\beta$ in subjects with a BMI of less than $25 \mathrm{~kg} / \mathrm{m}^{2}(\mathrm{r}=-0.46, \mathrm{p}<$ $0.05, \mathrm{r}=-0.58, \mathrm{p}<0.01)$. Inverse correlations were observed between the level of uric acid and the CT HU values of the iliopsoas muscle and the rectus abdominis muscle in subjects with a BMI ranging from 25 to $30 \mathrm{~kg} / \mathrm{m}^{2}$ ( $\mathrm{r}=-0.33$, $\mathrm{p}<$ $0.05, \mathrm{r}=-0.35, \mathrm{p}<0.01)$. Positive correlations were observed between the level of ALT and the CT HU values of the iliopsoas muscle, back muscle and rectus abdominis muscle in subjects with a BMI ranging from 25 to $30 \mathrm{~kg} / \mathrm{m}^{2}$ ( $\mathrm{r}=$ $0.29, \mathrm{r}=0.28$ and $\mathrm{r}=0.29$, respectively; $\mathrm{p}<0.05$ ). Positive correlations were observed between the level of triglycerides and the CT HU values of the back muscle and rectus abdominis muscle in subjects with a BMI higher than $30 \mathrm{~kg} /$ $\mathrm{m}^{2}(\mathrm{r}=0.45, \mathrm{p}<0.01 ; \mathrm{r}=0.40, \mathrm{p}<0.05)$.

Of note, inverse correlations were observed between the liver CT HU value and the levels of liver enzymes (AST and ALT) ( $r=-0.42$ and $r=-0.49)$ and glucose-lipid metabolic markers (fasting insulin, HOMA-IR, HOMA- $\beta$ and glycosylated hemoglobin) $(\mathrm{r}=-0.52, \mathrm{r}=-0.51, \mathrm{r}=-0.41$ and $\mathrm{r}=-0.30$, respectively; $\mathrm{p}<0.01$ ), confirming that lipid deposition in the liver is indicative of hepatic dysfunction and glucose dyshomeostasis.

In a multiple regression analysis, the $\mathrm{CT} H \mathrm{HU}$ values of the three kinds of skeletal muscles were found to have no significant impact on the levels of glucose-lipid metabolic markers (Table 3).

\section{Study 2}

\section{Group A (Lifestyle modification)}

A total of 28 men (age: $55 \pm 11.4$ years; body weight: $76.0 \pm 10.2 \mathrm{~kg}$; BMI: $27.1 \pm 3.5 \mathrm{~kg} / \mathrm{m}^{2}$; waist circumference: $94.4 \pm 6.1 \mathrm{~cm}$ ) were included in this study. All participants adopted lifestyle modifications, including walking (approximately 10,000 steps per day or approximately one hour per day for three to five days per week) and a specific diet (25 $\mathrm{kcaL} / \mathrm{kg}+0 \sim 200 \mathrm{kcaL}$ ), for three months. After this threemonth period, significant decreases in body weight (from $76.0 \pm 10.2$ to $74.0 \pm 10.0 \mathrm{~kg}, \mathrm{p}<0.01$ ), BMI (from $27.1 \pm 3.5$ to $26.7 \pm 3.6 \mathrm{~kg} / \mathrm{m}^{2}, \mathrm{p}<0.01$ ) and waist circumference (from $94.4 \pm 6.1$ to $92.3 \pm 8.2 \mathrm{~cm}, \mathrm{p}<0.01)$ were observed. The VFA values also significantly decreased (from 187.0 \pm 55.3 to $\left.174.0 \pm 58.8 \mathrm{~cm}^{2}, \mathrm{p}<0.01\right)$. On the other hand, no significant changes were observed in SFA. After the interventions, significant increases in the CT HU values of the iliopsoas muscle (from $43.0 \pm 4.6$ to $46.2 \pm 4.9 \mathrm{HU}, \mathrm{p}<0.01$ ) and back muscle (from $40.0 \pm 9.3$ to $42.8 \pm 9.6 \mathrm{HU}, \mathrm{p}<0.01$ ) were detected. In contrast, no changes were observed in the CT HU values of the rectus abdominis muscle. Although the differences were not significant, there was a trend toward improvement in the CT HU values of the liver (from $51.7 \pm 11$ to $55.0 \pm 8.8$ $\mathrm{HU}, \mathrm{p}=0.4)$. No changes were observed in the tested fuel metabolism markers. 
Table 3. Potential Associations between the CT HU Values of Each Tissue and the Levels of Glucose-lipid Metabolic Markers in a Multiple Regression Analysis

\begin{tabular}{|c|c|c|c|c|c|c|c|c|}
\hline & \multicolumn{8}{|c|}{ Predictor variable } \\
\hline & $\begin{array}{l}\text { CT HU value of } \\
\text { iliopsoas muscle }\end{array}$ & $\begin{array}{l}\text { CT HU value of } \\
\text { back muscle }\end{array}$ & $\begin{array}{l}\mathrm{CT} \mathrm{HU} \text { value of } \\
\text { rectus abdominis } \\
\text { muscle }\end{array}$ & & $\begin{array}{l}\text { CT HU value of } \\
\text { liver }\end{array}$ & & VFA & \\
\hline & F value & F value & F value & & F value & & F value & \\
\hline FPG (mg/dL) & 0.459986 & 1.514525 & 0.310999 & & 0.09517 & & 0.022875 & \\
\hline Fasting insulin $(\mu \mathrm{U} / \mathrm{mL})$ & 0.016163 & 0.269931 & 0.372145 & & 28.75759 & $* *$ & 6.411355 & $* *$ \\
\hline HOMA-IR & 0.024614 & 0.19633 & 1.271732 & & 26.29663 & $* *$ & 8.971085 & $* *$ \\
\hline НОМА- $\beta$ & 1.337558 & 2.450484 & 0.104022 & & 12.38965 & $* *$ & 4.258011 & $*$ \\
\hline HbAlc (\%) & 0.000122 & 0.055566 & 0.343369 & & 0.786111 & & 0.738849 & \\
\hline T-Cho (mg/dL) & 2.807251 & 0.52064 & 2.110077 & & 0.6234 & & 0.702938 & \\
\hline HDL-C (mg/dL) & 0.480973 & 0.005649 & 0.741931 & & 0.042927 & & 0.376761 & \\
\hline $\mathrm{TG}(\mathrm{mg} / \mathrm{dL})$ & 0.046872 & 0.069886 & 0.042644 & & 0.516454 & & 0.361545 & \\
\hline AST (IU/L) & 0.056663 & 0.296184 & 5.598727 & $*$ & 0.000453 & & 0.710747 & \\
\hline ALT (IU/L) & 0.285036 & 0.417567 & 2.133421 & & 0.799271 & & 1.506195 & \\
\hline$\gamma$-GTP (IU/L) & 0.136143 & 0.56958 & 0.020191 & & 0.506694 & & 0.059505 & \\
\hline FFA (mmol/L) & 0.679677 & 0.034012 & 0.161059 & & 0.148468 & & 0.020256 & \\
\hline
\end{tabular}

$* \mathrm{p}<0.05, * * \mathrm{p}<0.01$

VFA: Visceral fat area, FPG: Fasting plasma glucose, HDL-C: High-density lipoprotein cholesterol, TG: Triglyceride, AST: Aspartate 2-oxoglutarate aminotransferase, ALT: Alanine 2-oxoglutarate aminotransferase, HbA1c: Glycosylated hemoglobin, T-Cho: Total cholesterol, FFA: Free fatty acid, $\gamma$-GTP: Gamma-glutamyl transpeptidase

\section{Group B (Pioglitazone treatment)}

A total of 24 men (age: $55 \pm 9.0$ years; body weight: $74.0 \pm$ $12.1 \mathrm{~kg}$; BMI: $27.0 \pm 3.9 \mathrm{~kg} / \mathrm{m}^{2}$; waist circumference: $93.9 \pm$ $8.7 \mathrm{~cm})$ were included. The subjects were treated with Pioglitazone (30 mg/day) for three months. After treatment, no changes were observed in body weight, BMI or waist circumference. A significant decrease was observed in VFA (from $200.0 \pm 61.0$ to $176.0 \pm 63.1 \mathrm{~cm}^{2}, \mathrm{p}<0.01$ ). In contrast, no significant changes were detected in SFA. No changes were observed in the CT HU values of the iliopsoas muscle, back muscle or rectus abdominis muscle. At the end of treatment, significant increases in the CT HU values of the liver were observed (from $52.0 \pm 11.5$ to $57.3 \pm 8.9 \mathrm{HU}, \mathrm{p}<$ 0.01) (Fig. 3) accompanied by significant improvements in the liver function. Significant decreases were also observed in the levels of FPG (from $122.0 \pm 18.8$ to $115.6 \pm 19.4 \mathrm{mg}$ / $\mathrm{dL}, \mathrm{p}<0.05$ ), fasting insulin (from $12.0 \pm 8.6$ to $9.0 \pm 5.3 \mu \mathrm{U} /$ $\mathrm{mL}, \mathrm{p}<0.01$ ), HOMA-IR (from $3.6 \pm 2.2$ to $2.6 \pm 2.0, \mathrm{p}<0.01$ ) and triglycerides (from $257.3 \pm 353.5$ to $142.7 \pm 59.7 \mathrm{mg} / \mathrm{dL}$, p $<0.05)$.

\section{Group C (Miglitol treatment)}

A total of 16 men (age: $58 \pm 9.7$ years; body weight: $76.0 \pm$ $8.0 \mathrm{~kg}$; BMI: $28.5 \pm 2.7 \mathrm{~kg} / \mathrm{m}^{2}$; waist circumference: $94.3 \pm 5.9$ $\mathrm{cm}$ ) were included in this section of the study. The participants were treated with Miglitol (150 mg/day) for three months. At the end of the treatment period, significant decreases in body weight (from $76.0 \pm 8.0$ to $73.0 \pm 7.1 \mathrm{~kg}, \mathrm{p}<$ 0.01 ), BMI (from $28.5 \pm 2.7$ to $27.3 \pm 2.3 \mathrm{~kg} / \mathrm{m}^{2}, \mathrm{p}<0.01$ ) and waist circumference (from $94.3 \pm 5.9$ to $90.7 \pm 4.9 \mathrm{~cm}, \mathrm{p}<0.01$ ) were observed. Significant decreases in VFA (from 207.0 \pm 48.2 to $171.0 \pm 40.3 \mathrm{~cm}^{2}, \mathrm{p}<0.01$ ) and SFA (from 171.0 \pm 46.5 to $\left.152.0 \pm 37.9 \mathrm{~cm}^{2}, \mathrm{p}<0.05\right)$ were also observed. No changes in the CT values of the iliopsoas muscle, back muscle, rectus abdominis muscle or liver were observed (Fig. 3). Sig-

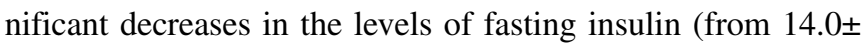
6.3 to $11.0 \pm 3.4 \mu \mathrm{U} / \mathrm{mL}, \mathrm{p}<0.05$ ) and HOMA-IR (from 4.0 \pm 1.9 to $3.1 \pm 1.2, \mathrm{p}<0.05)$ were observed. No changes were observed in the FPG levels or lipid metabolism.

\section{Potential associations between the changes in the CT HU values of the three skeletal muscles and changes in the other variables}

Due to the cross-sectional nature of the analysis, the associations do not necessarily indicate causality. Therefore, we attempted to investigate the potential associations between the changes in the CT HU values of the three skeletal muscles (delta value: 3 months (3M)-0 month (0M)) and changes in the other variables, including VFA (delta value: 3 months (3M)-0 month (0M)), during the observation period. The results were newly appended in Table 4. Overall, with the exception of some of the delta values in the metabolic parameters, the changes in the CT HU values of the three skeletal muscles did not exhibit any significant correlations with the changes in the other variables, including VFA.

\section{Discussion}

Using abdominal CT scan analyses, we herein attempted 
(a)

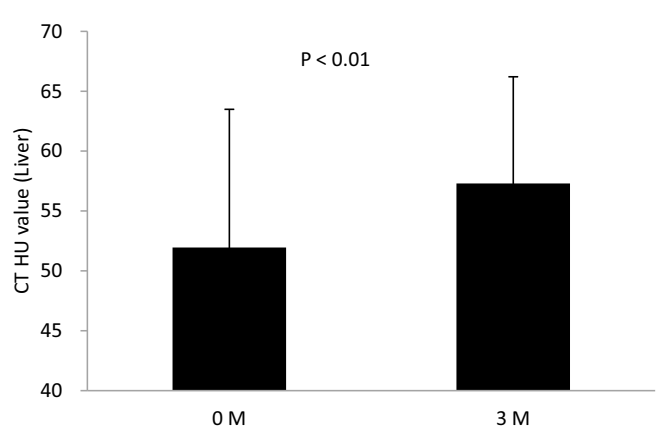

(c)

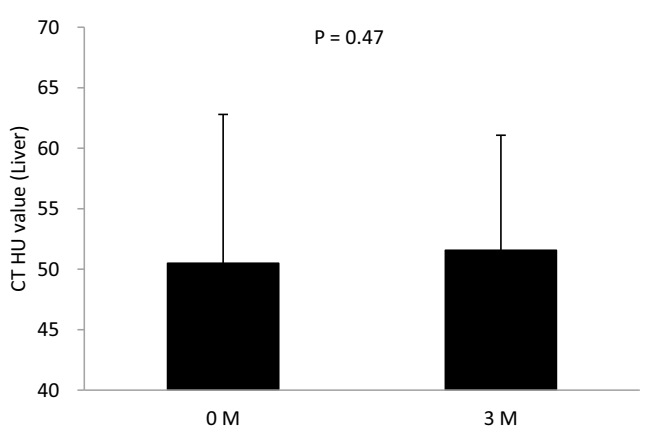

(b)

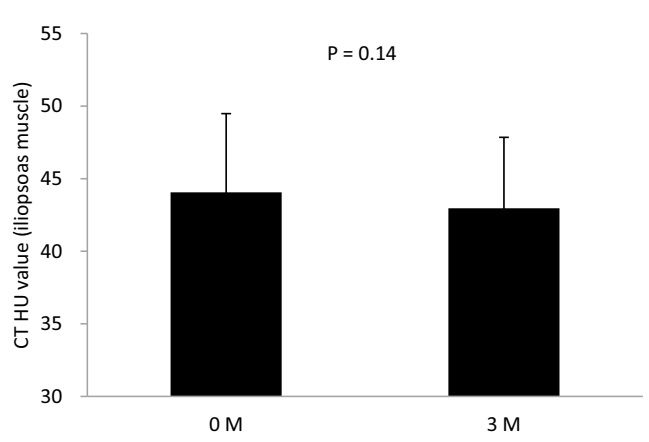

(d)

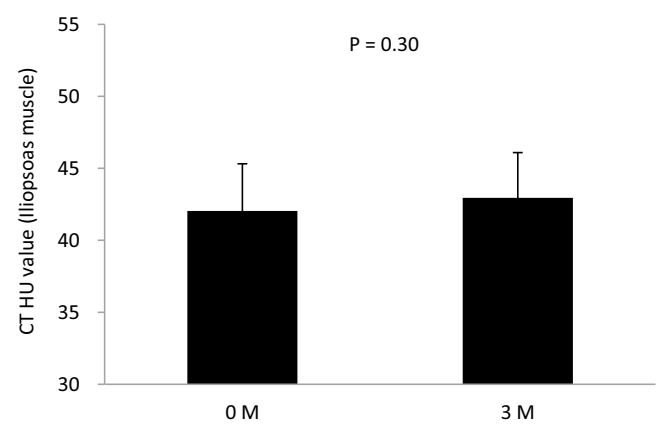

Figure 3. Changes in the CT HU values of the liver and skeletal muscles in the patients receiving pharmacological treatments. (a) The Pioglitazone-treated patients $(n=24)$ exhibited significant increases in the CT HU values of the liver. (b) The Pioglitazone-treated patients $(n=24)$ exhibited no changes in the CT HU values of the skeletal muscles before and after the three-month treatment. (c) The Miglitol-treated patients $(n=16)$ exhibited no changes in the CT HU values of the liver before and after the three-month treatment. (d) The Miglitol-treated patients $(n=16)$ exhibited no changes in the CT HU values of the skeletal muscles before and after the three-month treatment.

to establish a concise, comprehensive evaluation method of assessing ectopic lipid deposition in conjunction with visceral fat accumulation. The major findings of the present study of middle-aged, mildly obese Japanese men are summarized as follows. In patients with metabolic syndrome, (1) visceral fat accumulation is correlated with lipid deposition in the iliopsoas muscle, back muscle, rectus abdominis muscle and liver, (2) lifestyle modifications and treatment with antidiabetic drugs, including Pioglitazone and Miglitol, significantly attenuate visceral fat accumulation and (3) liver lipid deposition is strongly correlated with biochemical markers of glucose-lipid metabolism.

A limited number of reports have explored the potential association between ectopic lipid deposition and visceral fat accumulation using the ${ }^{1} \mathrm{H}-\mathrm{MRS}$ method $(22,23)$. Recent studies using ${ }^{1} \mathrm{H}-\mathrm{MRS}$ in healthy, young populations have reported significant positive correlations (1) between the extent of intramyocellular lipid deposition in the soleus muscle and the VFA and (2) between the extent of intrahepatic lipid deposition and the VFA. Moreover, these studies reported positive correlations between the extent of intramyocellular lipid deposition in the soleus muscle and intrahepatic lipid deposition $(22,23)$. However, no correlations have been observed between the extent of intramyocellular lipid deposition in the tibialis anterior muscle and intrahepatic lipid deposition. Overall, there have been no reports addressing the association between visceral fat mass and skeletal muscle and liver lipid deposition in patients with metabolic syndrome. In this context, the present study demonstrated significant correlations (1) between the CT HU values of three different skeletal muscle lipid depots and the VFA and (2) between the CT HU value of the liver and the VFA. In contrast, there were no correlations between the CT HU values of the skeletal muscles and liver. In previous studies using ${ }^{1} \mathrm{H}-\mathrm{MRS}$, the intramyocellular lipid content was measured exclusively in the lower thigh in healthy, young populations $(22,23)$. In the present study, however, the skeletal muscle CT HU value was measured using the trunk muscles. Although the CT HU values of the liver and skeletal muscles may not necessarily reflect the intramyocellular and intrahepatic lipid content (24), the present study describes a concise, comprehensive method of evaluating ectopic lipid deposition that may be beneficial for assessing the metabolic status and the impact of therapy in patients with metabolic syndrome.

In the present study, lifestyle modifications caused significant decreases in lipid deposition in the iliopsoas and back muscles, whereas the amount of lipids in the rectus abdominis muscle did not change. Although the differences were not significant, there was a trend toward improvement in the 
Table 4. Associations between the Changes in the CT HU Values of the Three Skeletal Muscles and the Changes in the Other Variables during the Observation Period

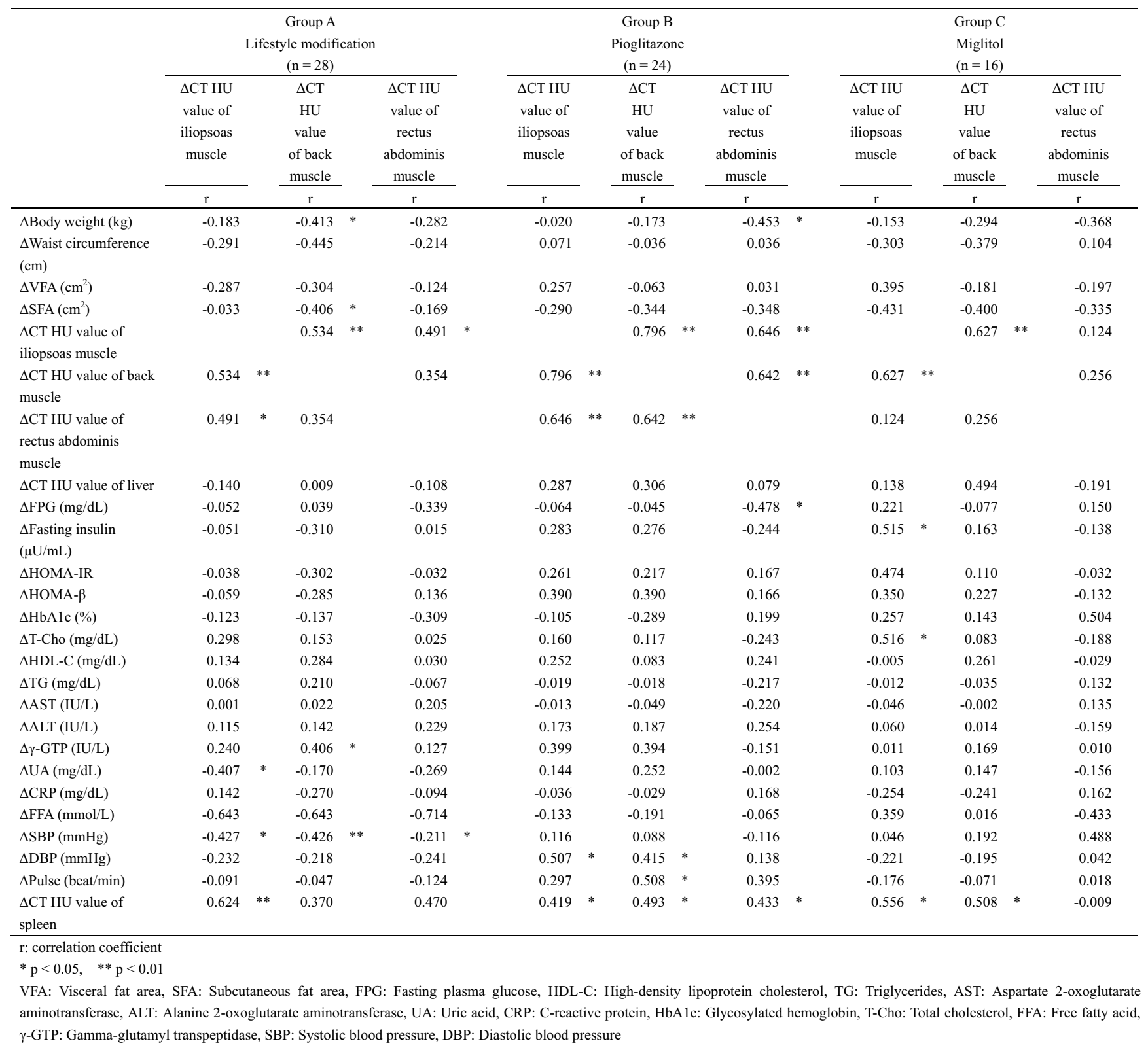

CT HU values of the liver. Noteworthy is the finding that the CT HU value of the rectus abdominis muscle exhibited significantly larger variation among individuals than the values of the iliopsoas and back muscles (Fig. 1). The greater interindividual variation observed in lipid deposition in the rectus abdominis may be linked to unique properties in the response to lifestyle modifications. Site-specific differences in muscle fiber components or sensitivity to stretch or exercise may also be involved. In this context, further studies are warranted to clarify which sites in human skeletal muscles are more susceptible to lipid deposition under conditions of overnutrition or decreased physical activity. Interestingly, in Study 1, a positive correlation was found between the iliopsoas muscle area and insulin resistance and an inverse correlation was observed between the back muscle area and the level of HDL-C. These findings suggest that the degree of muscular lipid deposition in each tissue is not necessarily correlated with muscular size.

The examination of the potential relationships between ectopic lipid deposition and metabolic parameters conducted in Study 1 revealed strong correlations between liver lipid deposition and the levels of glucose-lipid metabolic markers. Furthermore, treatment with Pioglitazone (Study 2) resulted in decreases in liver lipid deposition with concomitant improvements in the levels of glucose-lipid metabolic markers. These results are consistent with those of a previous study showing that the administration of Pioglitazone leads to improvement of hepatic insulin sensitivity and attenuation of liver lipid deposition in patients with nonalcoholic steatohepatitis (25). In this context, our data showing that Pioglitazone treatment improves insulin resistance and decreases lipid deposition in the liver validate the clinical setting of 
the present study. It is, therefore, tempting to speculate that liver lipid overload may have a greater impact on glucoselipid metabolic markers than skeletal muscle lipid overload.

The present study demonstrated that visceral and subcutaneous adipose tissue mass is reduced during treatment with Miglitol. On the other hand, ectopic lipid accumulation did not improve significantly in the patients treated with Miglitol. To our knowledge, there have so far been no reports addressing the mechanisms whereby Miglitol reduces visceral and subcutaneous adipose tissue mass (10). Further studies are warranted to clarify these underlying mechanisms.

The present study showed that lipid deposition in the iliopsoas muscle, back muscle and rectus abdominis muscle increases with age. This finding is in agreement with the results of a previous study that showed that age-related decreases in mitochondrial activity are closely associated with skeletal muscle lipid deposition (8). Furthermore, it is noteworthy that the extent of the decreases observed in lipid deposition in the skeletal muscles and liver exhibited wide variation among the three kinds of studies (Fig. 3). Therefore, it is possible that depot-specific susceptibility to lipid deposition exists. These findings lead to the speculation that changes in ectopic lipid deposition reflect responsiveness to antidiabetic drugs. Further studies are therefore warranted to test the hypothesis that depot-specific susceptibility to ectopic lipid deposition is associated with therapeutic effectiveness in patients with metabolic syndrome.

The use of proton ${ }^{1} \mathrm{H}-\mathrm{MRS}$ to evaluate tissue lipid content is not yet prevalent in clinics because the technique is expensive and elaborate. In line with this notion, we did not obtain our own data regarding a comparison between the MRS and CT HU values. However, a previous work reported that the liver fat content, as assessed by MRS, in patients with nonalcoholic fatty liver disease exhibits good correlation with the CT HU values (26). Therefore, future studies are warranted to verify that such a correlation would also be reproduced in a variety of skeletal muscles in humans.

In summary, the present study is the first to show that visceral fat accumulation is correlated with lipid deposition in the iliopsoas muscle, back muscle, rectus abdominis muscle and liver in middle-aged men with metabolic syndrome. CT-based simultaneous evaluations of ectopic lipid deposition and visceral fat accumulation may provide unique information for assessing cardiometabolic risks and therapeutic impact.

\section{Study limitations}

There were some limitations associated with this study. First, we did not measure the tissue lipid content directly using ${ }^{1} \mathrm{H}-\mathrm{MRS}$. The CT HU values of the liver and skeletal muscles may not necessarily reflect the intramyocellular or intrahepatocellular lipid content, although they do reflect the extent of relative lipid deposition in each tissue.

Second, the correlations between the CT HU values of the truncal muscles and body weight, waist circumference, the levels of hepatic enzymes and the levels of glucose-lipid metabolic markers were negligible. Therefore, it remains unclear in the present study whether changes in the CT HU values of the truncal muscles reflect whole body glucoselipid metabolism.

Third, there is a limitation of a $\beta$ error (type 2 error) in our analysis due to the inclusion of 105 patients.

Indeed, we performed a post hoc power calculation to analyze whether the study was adequately powered to detect associations between these variables and the CT HU values of the three skeletal muscles. In our samples, given a total sample size of 105 at the $5 \%$ level of significance, the power (1- $\beta$ error probability) of the associations between the CT HU values of the iliopsoas, back and rectus abdominis muscles and the visceral fat area was calculated to be 0.78 , 0.99 and 0.99 , respectively, showing enough power to detect significant associations. Meanwhile, the power of the associations between the CT HU values of the iliopsoas, back and rectus abdominis muscles and the levels of FPG, fasting insulin, HOMA-IR, HOMA- $\beta$, HDL-C, total cholesterol (TCho), triglyceride (TG), AST, ALT, glycosylated hemoglobin (HbA1c), FFA and $\gamma$-GTP in our 105 samples was quite low (power $=0.05$ to 0.07 ), except for the association between the CT HU value of the rectus abdominis muscle and the level of AST (power=0.79). Since at least 200 subjects are required to detect mild associations ( $r>0.20)$ with $80 \%$ power at the $5 \%$ level of significance, there is a limitation to commit a $\beta$ error (type 2 error) in our analysis.

The authors state that they have no Conflict of Interest (COI).

\section{Acknowledgement}

This work was supported in part by the Special Account Budget for Education and Research granted by the MEXT JAPAN, The Takeda Science Foundation, Specified Research Grant, Grants-in-Aid (MEXT, JAPAN) (Category C) (No.22591014 and No. 225921059), the Okinawa Medical Science Research Foundation and a Research Promotion Grant of the University of the Ryukyus Foundation and the project to "Establish a research hub toward the development of an intellectual cluster," Okinawa Prefecture, Japan.

M.S. and H.M. designed the studies. S.T., M.S., M.H., K.Y., C.K., R.U., S.S., M.D., T.N., E.K., Y.N., H.N., T.I., S.N., T.T., T. I. and K.Y. conducted the data collection. S.T., M.S., M.H., K.Y., C.K., R.U., S.S., Y.O., K.Y. and H.M. contributed to the discussion. S.T. and H.M. wrote, reviewed and edited the manuscript.

We are grateful to Ms. N. Yamamoto, M. Katayanagi, M. Hirata, I. Asato and C. Noguchi for their secretarial assistance. We also thank Ms. K. Iwabuchi, K. Nakamoto and A. Ohshiro for their technical assistance.

\section{References}

1. Masuzaki H, Paterson J, Shinyama H, et al. A transgenic model of visceral obesity and the metabolic syndrome. Science 294: 21662170, 2001.

2. Masuzaki H, Yamamoto H, Kenyon CJ, et al. Transgenic amplification of glucocorticoid action in adipose tissue causes high blood 
pressure in mice. J. Clin. Invest 112: 83-90, 2003.

3. Despres JP, Lemieux I. Abdominal obesity and metabolic syndrome. Nature 444: 881-887, 2006.

4. Mandavilli A, Cyranoski D. Asia's big problem. Nature Med 10 325-327, 2004.

5. Montague CT, O'Rahilly S. The perils of portliness: causes and consequences of visceral adiposity. Diabetes 49: 883-888, 2000.

6. Shimabukuro M, Zhou YT, Lee Y, Unger RH. Troglitazone lowers islet fat and restores beta cell function of Zucker diabetic fatty rats. J Biol Chem 273: 3547-3550, 1998.

7. Wang MY, Grayburn P, Chen S, Ravazzola M, Orci L, Unger RH. Adipogenic capacity and the susceptibility to type 2 diabetes and metabolic syndrome. Proc Natl Acad Sci USA 105: 6139-6144, 2008.

8. Peterson KF, Befroy D, Dufour S, et al. Mitochondrial dysfunction in the elderly: possible role in insulin resistance. Science $\mathbf{3 0 0}$ : 1140-1142, 2003.

9. Tamura Y, Tanaka Y, Sato F, et al. Effects of diet and exercise on muscle and liver intracellular lipid contents and insulin sensitivity in type 2 diabetic patients. J Clin Endocrinol Metab 90: 31913196, 2005.

10. Shimabukuro M, Higa M, Yamakawa K, Masuzaki H, Sata M. Miglitol, $\alpha$-glycosidase inhibitor, reduces visceral fat accumulation and cardiovascular risk factors in subjects with the metabolic syndrome: A randomized comparable study. Int J Cardiol 2012 (in press).

11. Matsuzawa Y. Metabolic syndrome-definition and diagnostic criteria in Japan. J Atheroscler Thromb 12: 301, 2005.

12. Arai H, Yamamoto A, Matsuzawa $Y$, et al. Prevalence of metabolic syndrome in the general Japanese population in 2000. J Atheroscler Thromb 13: 202-208, 2006.

13. Alberti KG, Zimmet P, Shaw J; IDF Epidemiology Task Force Consensus Group. The metabolic syndrome-a new worldwide definition. Lancet 366: 1059-1062, 2005.

14. Shimabukuro M. Cardiac adiposity and global cardiometabolic risk: new concept and clinical implication. Circ J 73: 27-34, 2009.

15. Seino Y, Nanjo K, Tajima N, et al. Report of the committee on the classification and diagnostic criteria of diabetes mellitus. Diabetol
Int 1: 2-20, 2010.

16. Hadar H, Gadoth N, Heifetz M. Fatty replacement of lower paraspinal muscles: normal and neuromuscular disorders. Am J Roentgenol 141: 895-898, 1983.

17. Kelley DE, Slasky BS, Janosky J. Skeletal muscle density: effects of obesity and non-insulin-dependent diabetes mellitus. Am J Clin Nutr 54: 509-515, 1991.

18. Hawley RJ Jr, Schellinger D, O'Doherty DS. Computed tomographic patterns of muscles in neuromuscular diseases. Arch Neurol 41: 383-387, 1984.

19. Goodpaster BH, Thaete FL, Kelley DE. Thigh adipose tissue distribution is associated with insulin resistance in obesity and in type 2 diabetes mellitus. Am J Clin Nutr 71: 885-892, 2000.

20. Hamer OW, Aguirre DA, Casola G, Lavine JE, Woenckhaus M, Sirlin CB. Fatty Liver: imaging patterns and pitfalls. Radiographics 26: 1637-1653, 2006.

21. Speliotes EK, Massaro JM, Hoffmann U, et al. Liver fat is reproducibly measured using computed tomography in the Framingham Heart Study. J Gastroenterol Hepatol 23: 894-899, 2008.

22. Hwang JH, Stein DT, Barzilai N, et al. Increased intrahepatic triglyceride is associated with peripheral insulin resistance: in vivo MR imaging and spectroscopy studies. Am J Physiol Endocrinol Metab 293: E1663-E1669, 2007.

23. Larson-Meyer DE, Newcomer BR, Ravussin E, et al. Intrahepatic and intramyocellular lipids are determinants of insulin resistance in prepubertal children. Diabetologia 54: 869-875, 2011.

24. Goodpaster BH, Kelley DE, Thaete FL, He J, Ross R. Skeletal muscle attenuation determined by computed tomography is associated with skeletal muscle lipid content. J Appl Physiol 89: 104110, 2000.

25. Belfort R, Harrison SA, Brown $\mathrm{K}$, et al. A placebo-controlled trial of pioglitazone in subjects with nonalcoholic steatohepatitis. N Engl J Med 355: 2297-2307, 2006.

26. Zhong L, Chen JJ, Chen J, et al. Nonalcoholic fatty liver disease: quantitative assessment of liver fat content by computed tomography, magnetic resonance imaging and proton magnetic resonance spectroscopy. J Dig Dis 10: 315-320, 2009.

(C) 2013 The Japanese Society of Internal Medicine http://www.naika.or.jp/imonline/index.html 